\title{
Estimación del atributo satisfacción en test de usuarios a partir del análisis de la expresión facial ${ }^{*}$
}

\author{
Darly Mildred Delgado Agudelo** \\ Diego Fernando Girón Timaná*** \\ Gabriel Elías Chanchí Golondrino**** \\ Katerine Márceles Villalba $a^{* * * *}$
}

\author{
Recibido: 18/10/2018 • Aceptado: 28/06/2019 \\ https://doi.org/10.22395/rium.v19n36a1
}

\begin{abstract}
Resumen
La usabilidad se ha convertido en un aspecto fundamental en el desarrollo de un producto software por ser un atributo primordial de la calidad. Este concepto está ligado al carácter de uso del software y puede ser evaluado a través de una prueba de usabilidad. Dicha evaluación, se realiza de manera cuantitativa mediante métricas para dos de los atributos (eficacia y eficiencia) que la definen. La satisfacción como tercer atributo asociado es de carácter cualitativo, cuya estimación es subjetiva, pues depende de la ausencia de incomodidad del usuario de prueba durante la interacción con el producto. Este artículo es resultado de una investigación que postula una propuesta para la estimación de la satisfacción en test de usuarios, teniendo en cuenta el análisis de la expresión facial. Esta idea está soportada en el desarrollo de un sistema software para el seguimiento emocional de un usuario durante una prueba.
\end{abstract}

Palabras clave: expresión facial; satisfacción; test de usuario; usabilidad.

\footnotetext{
* Artículo derivado del proyecto de investigación de convocatoria interna Propuesta de método automatizado para la medición de la satisfacción en pruebas de usabilidad a partir del estudio de la expresión facial. Institución que financia: Institución Universitaria Colegio Mayor del Cauca. Ejecución: 12 de febrero de 2018 al 12 de febrero de 2019.

** Ingeniera en Informática, Institución Universitaria Colegio Mayor del Cauca, Popayán, Colombia. Correo electrónico: darlydelgado@unimayor.edu.co. Orcid: http://orcid.org/0000-0002-8405-2697

*** Ingeniero en Informática, Institución Universitaria Colegio Mayor del Cauca, Popayán, Colombia. Correo electrónico: dfgiron@unimayor.edu.co. Orcid: http://orcid.org/0000-0002-0550-4721

**** Doctor en Ingeniería Telemática. Profesor Facultad de Ingeniería de la Universidad de Cartagena, Cartagena de Indias, Bolívar, Colombia. Correo electrónico: gchanchig@unicartagena.edu.co. Orcid: http://orcid. org/0000-0002-0257-1988

****** Magíster en Seguridad Informática. Grupo de Investigación I+D en Informática, Facultad de Ingeniería, Institución Universitaria Colegio Mayor del Cauca, Popayán, Colombia. Correo electrónico: kmarceles@unimayor. edu.co. Orcid: http://orcid.org/0000-0002-4571-0714
} 


\title{
Estimate of the satisfaction attribute in user tests from facial expression analysis
}

\begin{abstract}
Usability has become a fundamental aspect of the development of a software product for being a primordial feature of quality. This concept is linked to the use nature of software and can be evaluated through a usability test. This testing is made quantitatively through measurement for two of the features (efficacy and efficiency) that define it. The satisfaction as a third associated feature is of a qualitative kind which estimation is subjective given that it depends on the absence of discomfort by the test user during its interaction with the product. This article is the result of a research that proposes an approach for the estimation of the satisfaction in user tests involving facial expression analysis. This idea is supported in the development of a software system for the emotional monitoring of a user during a test.
\end{abstract}

Keywords: facial expression; satisfaction; user test; usability.

\section{Estimativa do atributo "satisfação" em teste de usuários a partir da análise da expressão facial}

\begin{abstract}
Resumo
A usabilidade tem se tornado um aspecto fundamental no desenvolvimento de um produto software por ser um atributo primordial da qualidade. Esse conceito está ligado ao caráter de uso do software e pode ser avaliado por meio de um teste de usabilidade. Essa avaliação é realizada de maneira quantitativa, mediante métricas para dois dos atributos (eficácia e eficiência) que a definem. A satisfação como terceiro atributo associado é de caráter qualitativo, cuja estimativa é subjetiva, pois depende da ausência de desconforto do usuário do teste durante a interação com o produto. Este artigo é resultado de uma pesquisa que apresenta uma proposta para estimar a satisfação em teste de usuários, considerando a análise da expressão facial. Essa ideia está apoiada no desenvolvimento de um sistema software para o seguimento emocional de um usuário durante o teste.
\end{abstract}

Palavras-chave: expressão facial; satisfação; teste de usuário; usabilidade. 


\section{INTRODUCCIÓN}

El auge de la tecnología ha traído consigo propuestas novedosas creadas a partir de nuevos campos donde el desarrollo de software presenta avances significativos como la visión y la inteligencia artificial, la robótica, el internet de las cosas, entre otros adelantos, lo que ha permitido que la demanda de estas tecnologías aplicadas crezca exponencialmente en un sin número de dispositivos y con ello la exigencia en la calidad del software de estos aplicativos aumente [1]. Debido a esto, el concepto de usabilidad se ha convertido en un asunto esencial para el desarrollo informático, puesto que define el grado de uso que estas nuevas tecnologías proveen al consumidor final [2].

De acuerdo a la norma ISO 9241-11, la usabilidad es definida como: "el grado en que un producto puede ser usado por determinados usuarios para lograr sus propósitos con eficacia, eficiencia y satisfacción en un contexto de uso específico" [3]. Esta definición describe los tres atributos (eficiencia, eficacia y satisfacción) que se deben tener en cuenta para estimar el grado de usabilidad de un producto software. En una prueba de usuario, realizada dentro del contexto técnico de un laboratorio de usabilidad, la eficacia puede ser obtenida a partir del porcentaje de tareas ejecutadas debidamente por el usuario de prueba escogido. En lo que respecta a la eficiencia, puede ser medida por medio del tiempo empleado para realizar las tareas establecidas por el evaluador de la prueba [4].

Por su parte, la norma ISO 9241-11 define a la satisfacción como: “ausencia de incomodidad y existencia de actitudes positivas hacia la utilización del producto" [3]. Por otro lado, según la ISO 9126-1, la satisfacción es definida como la capacidad del software para cumplir con las expectativas del usuario en un contexto de uso determinado [7], relacionada directamente con la emocionalidad del usuario durante una prueba de usabilidad. Considerando esto en una prueba de usabilidad, la satisfacción se estima generalmente a partir de encuestas de percepción realizadas después de la prueba o mediante la observación del comportamiento del usuario, es decir, a través del análisis de los gestos, posturas y expresiones faciales evidenciadas durante la interacción con el software evaluado [4].

Todo esto se lleva a cabo en un laboratorio de usabilidad con las herramientas necesarias mediante diferentes test de usuario [2,7]. El primero es definido como un espacio físico compuesto de un área para el usuario y otro para los evaluadores, donde se realizan evaluaciones de usabilidad y accesibilidad a productos, sistemas y dispositivos por medio de software y equipos hardware especializados en el monitoreo, entre otros que registren el accionar del sujeto de prueba con el aplicativo a evaluar, teniendo en cuenta que deben contar con herramientas que permitan obtener indicadores 
de los atributos de eficiencia, eficacia y satisfacción [6]. Por su parte, el test de usuario es una técnica en el diseño y desarrollo de aplicaciones software y productos, para evaluar su grado de uso mediante la inspección in situ de la interacción del usuario con la herramienta. Los resultados de esta prueba son inmediatos y su principal característica es que el sujeto de prueba se encuentre aislado, pero monitoreado por los evaluadores.

En consecuencia, la obtención de la satisfacción es un proceso con cierto grado de subjetividad, pues depende en gran medida de la percepción del coordinador de la prueba con respecto al comportamiento del usuario, lo que no permite parametrizar cuantitativamente este atributo, algo que sí es posible con los de eficiencia y eficacia [5].

Por esta subjetividad en la obtención del atributo satisfacción, este artículo plantea una propuesta para su estimación en pruebas de usabilidad, tomando como variable de análisis la expresión facial de usuario. Esto teniendo en cuenta que la expresión facial es una de las variables menos invasivas y más usadas en un laboratorio de usabilidad, en comparación con el uso de diversos sensores fisiológicos que se articulan al espacio corporal del usuario.

Ahora, para garantizar la claridad de este concepto, es necesario indicar que proviene de las emociones y diferentes estados anímicos. Tenemos que las emociones tienen origen a nivel fisiológico en el sistema límbico (sistema cuya función es la de regular las emociones, la memoria, el hambre e instintos sexuales y se compone de partes del cerebro como el tálamo, el hipotálamo y la amígdala cerebral) del cuerpo humano y que crean estos estados [9].

Entonces, para evaluar o estimar las emociones observables en la expresión facial, ha sido necesario desarrollar tecnologías biométricas [10, 11], lo que permite su monitoreo y seguimiento por medio de instrumentos como cámaras web o sensores, que perciben las permutaciones fisiológicas derivadas de las emociones que se transmiten en las terminaciones nerviosas, el sudor de la piel o las expresiones faciales [12]; aunque estas tienen características novedosas, muchas son de difícil manejo en escenarios tan invasivos como el de la evaluación de usabilidad.

Las expresiones faciales son parte de la comunicación no verbal que genera el cuerpo, la captura de emociones consiste en utilizar estándares y patrones que permitan identificar por medio de estas expresiones los distintos estados emocionales, dado que los seres humanos no realizan las mismas expresiones 
faciales para transmitir siempre determinada emoción. Por ejemplo, el enojo frente a una situación puede tener la misma reacción facial que una expresión de incertidumbre, debido a esto se utiliza un modelo de emociones para seleccionar la más acorde a la información que se ha capturado del rostro [13]. Por esta razón la captura de emociones y expresiones faciales debe realizarse en tiempo real por parte del coordinador de la prueba.

Así mismo, el uso de la expresión facial está relacionado con el aprovechamiento y difusión de métodos computacionales que se vienen usando para la estimación de las emociones a partir de la expresión facial del usuario. La valoración de la satisfacción que propone este trabajo se realizó mediante el estudio emocional de la expresión facial con software automatizado para la estimación de la satisfacción en pruebas de usabilidad, teniendo como variable de entrada la expresión facial del usuario.

El software propuesto utiliza una cámara web dispuesta en un laboratorio de usabilidad (habitación del usuario), por medio de la cual se logra capturar imágenes continuas de su rostro para procesarlas mediante algoritmos de predicción de emociones (tristeza, felicidad, disgusto, sorpresa, neutralidad y enojo), tomando como base los modelos de clasificación de emociones de la librería OpenCV, los cuales infieren en tiempo real un estado emocional durante los diferentes momentos de la interacción. Una vez obtenidos los datos, el sistema software presenta gráficamente en tiempo real el comportamiento emocional de un usuario a lo largo de la prueba, así, el coordinador de esta puede discriminar la duración de las tareas y observar gráficamente dicho comportamiento por labor.

A diferencia de otras tecnologías para el reconocimiento facial, la herramienta propuesta se basa en tecnologías libres, esto permite la adecuación a las necesidades específicas de una prueba de usabilidad, y es apoyo para el coordinador de la prueba en cuanto a la estimación de la satisfacción. Así mismo, la herramienta no solo permite la detección del rostro sino también el reconocimiento de emociones durante la interacción, lo que es un elemento fundamental de cara a la obtención del atributo satisfacción como resultado de esta investigación.

\section{MATERIALES Y MÉTODOS}

El proceso investigativo inició con el abordaje de los modelos de emociones que son parametrizaciones de los estados emocionales del ser humano. Estos modelos son el resultado de investigaciones realizadas en la búsqueda de factores comunes en el comportamiento de un individuo. Debido a que las culturas son tan diversas, estas investigaciones han encontrado que las formas de expresar un sentimiento contienen 
expresiones universales que se aprecian por medio de cambios fisiológicos. Estas alteraciones generalmente se aprecian en los cambios de las expresiones faciales. El psicólogo y científico Paul Ekman investigó la manera de identificar trastornos mentales mediante la comunicación no verbal, específicamente en la expresión corporal.

Ekman pudo identificar diferentes expresiones faciales creadas por el movimiento de los músculos de la cara y logró darles significado como tipo de emoción. Además, en estos estudios se evidenció que las expresiones faciales son interculturales por lo que concretó una clasificación de expresiones faciales universales, identificando seis tipos de emociones primarias a saber: ira, asco, miedo, alegría, tristeza y sorpresa [14]. Para el desarrollo del sistema software propuesto se hizo uso de la librería de procesamiento de imágenes OpenCV, la cual realiza por defecto el procesamiento y detección de emociones sobre las imágenes capturadas, teniendo en cuenta una adaptación del modelo de Ekman (ver figura 1).

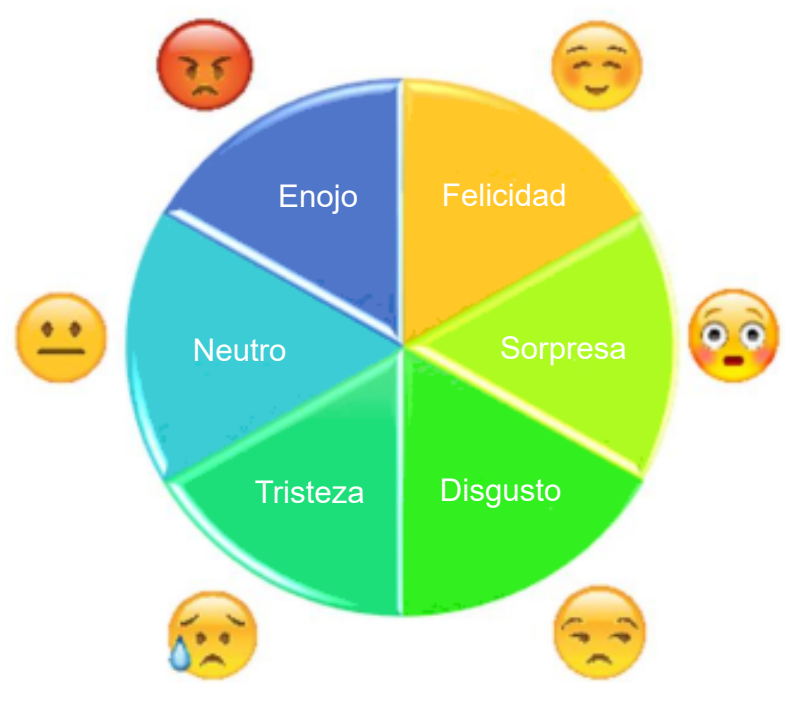

Figura 1. Modelo de emociones de Ekman Fuente: elaboración propia

Del modelo anterior se realizó un ajuste con relación a la emoción neutral, considerando que dicha emoción se logró identificar y no fue incluida dentro del modelo de Paul Ekman, por lo que los autores del artículo consideraron pertinente su inclusión.

Adicional al modelo Ekman, se consideraron un conjunto de trabajos para el desarrollo de la presente investigación. Estos tienen en cuenta el uso de modelos de emociones en diferentes contextos de aplicación. 
En [15], se describe el proyecto titulado Detección de emociones del usuario, donde se desarrolla un prototipo para la detección de emociones de usuario, analizándolas en tiempo real. La identificación de rostros e incongruencias en los datos obtenidos, condujo a la construcción de un modelo para la obtención de la matriz de proyección para cada sujeto. Este trabajo presenta como aporte el uso de la librería OpenCV para el procesamiento de imágenes y la propuesta de un modelo de reconocimiento de emociones.

Dentro de los antecedentes considerados, también se aplica el monitoreo de actividad cerebral, por ejemplo en [16] se propone una Interface cerebro-computador (Brain-computer interface - BCI) que permite la interacción de un usuario con el entorno cercano por medio de la actividad cerebral. Este tipo de tecnología crea un canal de comunicación para transmitir las intenciones, emociones, sensaciones, etc., de las personas a un dispositivo computarizado. $\mathrm{El} \mathrm{BCI}$ es un tipo de sistema que reconoce patrones en las señales del cerebro, mediante electroencefalografías capturadas por medio de sensores. La imagen arroja un patrón y el sistema determina un parámetro de satisfacción que puede ser clasificado en cuatro categorías dependiendo del estado mental del usuario: frustración, concentración y relajación. El trabajo tiene como falencia la manera intrusiva en la que se capturan los datos biométricos, ya que los sensores usados para registrar la actividad cerebral podrían causar una situación de incomodidad que puede distanciar el objetivo de medir la satisfacción de manera confiable [16].

Por su parte en [5] se presenta una propuesta para la estimación de la satisfacción en pruebas de usabilidad, a partir del análisis del estrés mental de un usuario durante el proceso de interacción. Esta propuesta realiza el estudio del estrés mental mediante el seguimiento de la variabilidad de la frecuencia cardiaca (VFC), la cual corresponde al espacio o conjunto de espacios entre pulsaciones cardiacas (intervalos RR). De este modo, partiendo de los intervalos RR y haciendo uso de la fórmula de Bayevsky [5] se obtiene el índice de estrés mental en diferentes momentos de la experiencia, lo que permite dar una idea general al coordinador de la prueba sobre el comportamiento emocional del usuario. Este trabajo presenta como aporte, la segmentación del proceso de estimación de la satisfacción de acuerdo con las tareas ejecutadas en la prueba, así como el seguimiento gráfico del estrés mental de un usuario [5].

De los anteriores trabajos se tuvieron en cuenta algunas consideraciones para la presente propuesta tales como: la presentación de los resultados mediante gráficos en tiempo real, el uso de dispositivos menos intrusivos para la obtención de variables fisiológicas que pueden usarse en la estimación de la satisfacción, como es el caso de la expresión facial, la consideración del modelo de emociones de Paul Ekman y el uso de la librería OpenCV para dar soporte al procesamiento y análisis emocional de las imágenes. 


\section{SISTEMA SOFTWARE PARA LA ESTIMACIÓN DE LA SATISFACCIÓN}

Aquí se describe el esquema funcional del sistema software propuesto (diagrama de bloques) y se muestran las interfaces gráficas finales del prototipo.

\subsection{Esquema funcional del sistema}

A continuación, se presenta el contexto de implementación del sistema software propuesto, el cual es un laboratorio de usabilidad.

Dentro del laboratorio de usabilidad, el equipo de desarrollo analizó los diferentes instrumentos utilizados en las pruebas de usuario convencionales y de manera específica el formato de seguimiento del coordinador de la prueba, discriminando las diferentes tareas que realiza el sujeto y anotando el comportamiento observado durante la misma (actitudes, gestos, expresiones faciales, etc.). En la tabla 1 se muestra el extracto de uno de los documentos de seguimiento usado por los evaluadores dentro del laboratorio de usabilidad de la Institución Universitaria Colegio Mayor del Cauca.

\begin{tabular}{|c|c|c|c|c|c|}
\hline Tarea & Descripción & Criterios de éxito & Cumplimiento & Tiempo & Observaciones \\
\hline & $\begin{array}{l}\text { 1. Abra el navegador Mozilla } \\
\text { Firefox e ingrese al portal }\end{array}$ & $\begin{array}{l}\text { Encuentra la sección: } \\
\text { "Lista de géneros" }\end{array}$ & & 10 & \\
\hline & $\begin{array}{l}\text { www.peliculashoy.com } \\
\text { 2. Encuentre la sección: "Lista } \\
\text { de géneros" y seleccione el }\end{array}$ & $\begin{array}{l}\text { Navega por el catálo- } \\
\text { go de películas y logra } \\
\text { encontrar la película } \\
\text { "21 Black Jack". }\end{array}$ & & & \\
\hline & $\begin{array}{l}\text { 3. Navegue por el catálogo de } \\
\text { películas y encuentre la pelí- } \\
\text { cula "21 Black Jack". } \\
\text { 4. Visualice la descripción de la } \\
\text { película seleccionada, y ano- } \\
\text { te la información solicitada. }\end{array}$ & $\begin{array}{l}\text { Visualiza la descrip- } \\
\text { ción de la película y } \\
\text { anota la información } \\
\text { solicitada. }\end{array}$ & & & \\
\hline
\end{tabular}

Tabla 1. Esquema funcional del sistema software

Fuente: elaboración propia

Así, teniendo en cuenta que un laboratorio de usabilidad consta del módulo de evaluador y usuario, donde para el segundo es que se disponen los bloques funcionales de captura de imagen, predicción de emociones, almacenamiento temporal, seguimiento en tiempo real, almacenamiento en base de datos, discriminación de tareas y generación gráfica de tareas (ver figura 2). 


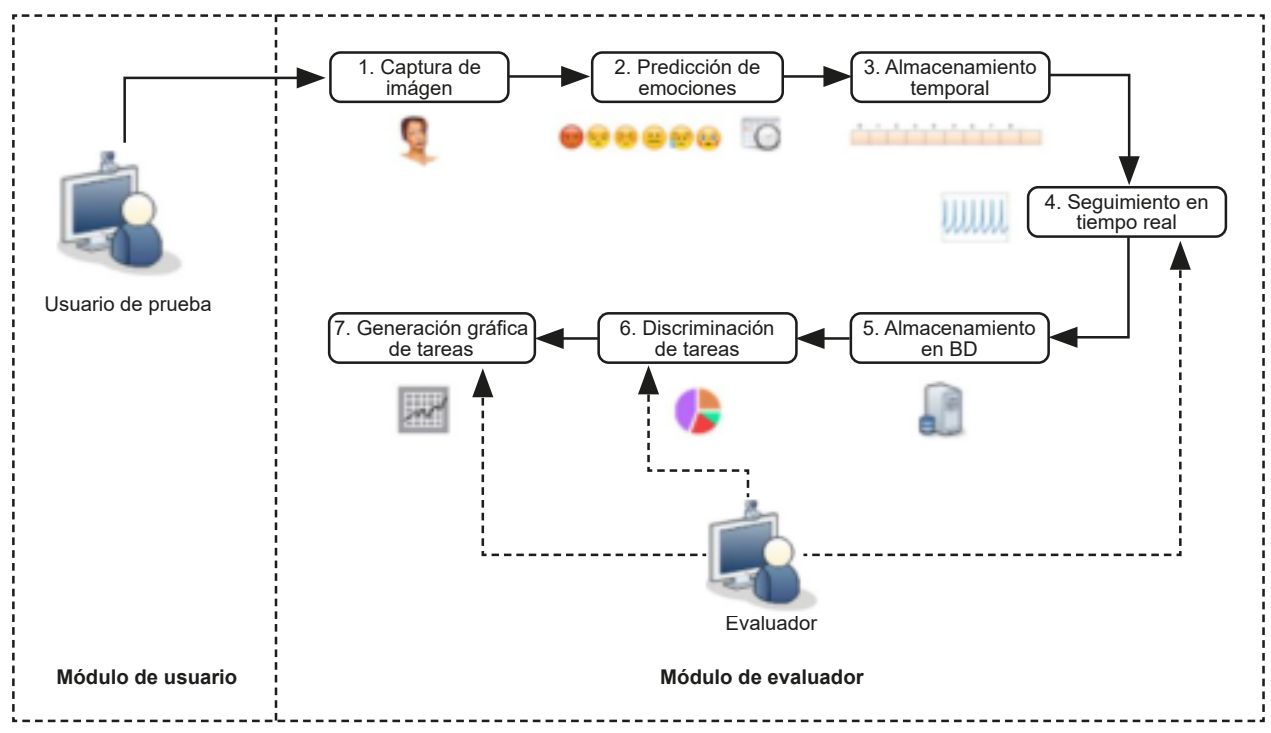

Figura 2. Esquema funcional del sistema software

Fuente: elaboración propia

Este último elemento de la discriminación de las tareas y su asociación con las emociones parte del modo en que se realiza el seguimiento de la satisfacción en pruebas de usuario.

Inicialmente en el módulo del usuario, se dispone la cámara web que enviará imágenes al bloque de captura de imagen, una vez el sistema software es ejecutado por parte del evaluador. Las imágenes tomadas al usuario durante la interacción de este con el aplicativo evaluado serán utilizadas para tomar registro del rostro y con esta información en el módulo de predicción de emociones, el sistema software hará uso de la librería OpenCV para validar y clasificar los rasgos capturados con el fin de obtener una de las posibles emociones del modelo: neutral, tristeza, felicidad, enojo, sorpresa y disgusto. El bloque de almacenamiento temporal es el encargado de almacenar en una lista la predicción de la emoción que ha sido validada junto a su tiempo de detección, para cotejar en el bloque de seguimiento en tiempo real, la descripción gráfica de la emoción capturada frente el tiempo en que fue detectada. En la parte de almacenamiento, el sistema software utilizando el gestor de bases de datos TinyDB guarda los registros (tiempo, emoción) de las capturas realizadas y graficadas en la etapa anterior. Consecutivamente, en el proceso de discriminación de tareas, el evaluador o coordinador de la prueba podrá ingresar numéricamente los rangos de tiempo expresados en segundos, para que el sistema software segmente en distintos tiempos la prueba, analizando la emoción capturada durante esos periodos. 
Finalmente, en el módulo de generación gráfica de tareas se muestran los datos obtenidos y el evaluador tendrá la posibilidad de exportar en formato CSV (commaseparated values), los resultados observados en el comportamiento emocional del usuario durante la prueba de usabilidad, para lo cual utiliza la librería Matplotlib de Python.

\subsection{Interfaces finales del sistema software}

En la figura 3, se muestra la interfaz principal del sistema software de estimación de satisfacción en pruebas de usabilidad. La interfaz permite incorporar el flujo de video de la cámara web del laboratorio de usabilidad y el recuadro de visualización en tiempo real de la gráfica de la emoción capturada y un botón que acciona o detiene el coordinador de la prueba cuando inicia o termina la prueba de usabilidad, así como también un botón de procesar que permitirá graficar el comportamiento emocional del usuario por tarea, un campo de texto llamado tarea donde se visualiza el tiempo de duración que tarda la prueba de usabilidad, otro campo llamado reporte donde se puede configurar un nombre de archivo para un posterior reporte y un botón de salir para dar por terminada la prueba. A continuación, se describe el funcionamiento del sistema software propuesto.

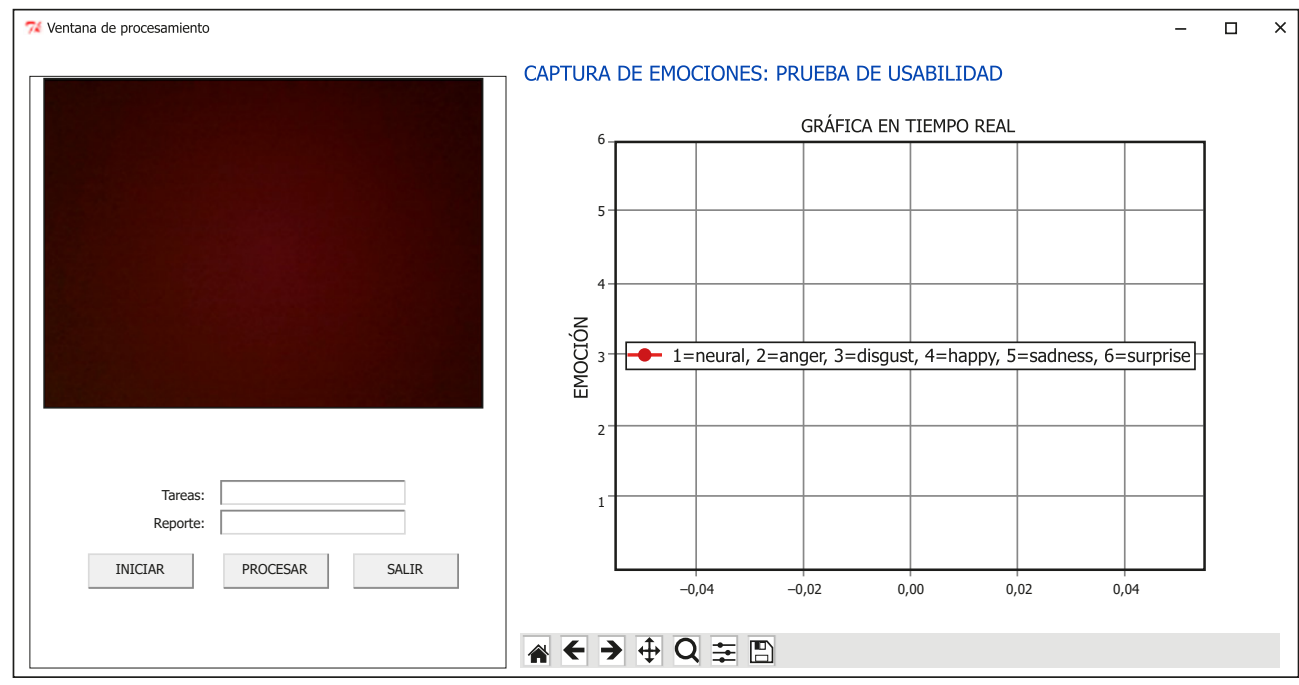

Figura 3. Interfaz principal del sistema software

Fuente: elaboración propia

Al momento que el usuario inicia la prueba de usabilidad dentro del laboratorio, el evaluador se encarga de accionar el botón para iniciar el sistema software, dando lugar al proceso de captura de imágenes del rostro usando una cámara de video o webcam. 
Inmediatamente se procede con la etapa de predicción, realizando un análisis de las imágenes capturadas con el fin de determinar una de las emociones correspondientes al modelo de clasificación usado por la librería OpenCV (ver figura 4), al tiempo que se muestra en el recuadro de visualización la emoción obtenida. El coordinador de la prueba es el encargado de indicarle al sistema software el momento en el que la prueba de usabilidad termina, de lo contrario este seguirá capturando las imágenes, realizando la predicción y mostrando gráficamente el comportamiento emocional del usuario a lo largo de la prueba.

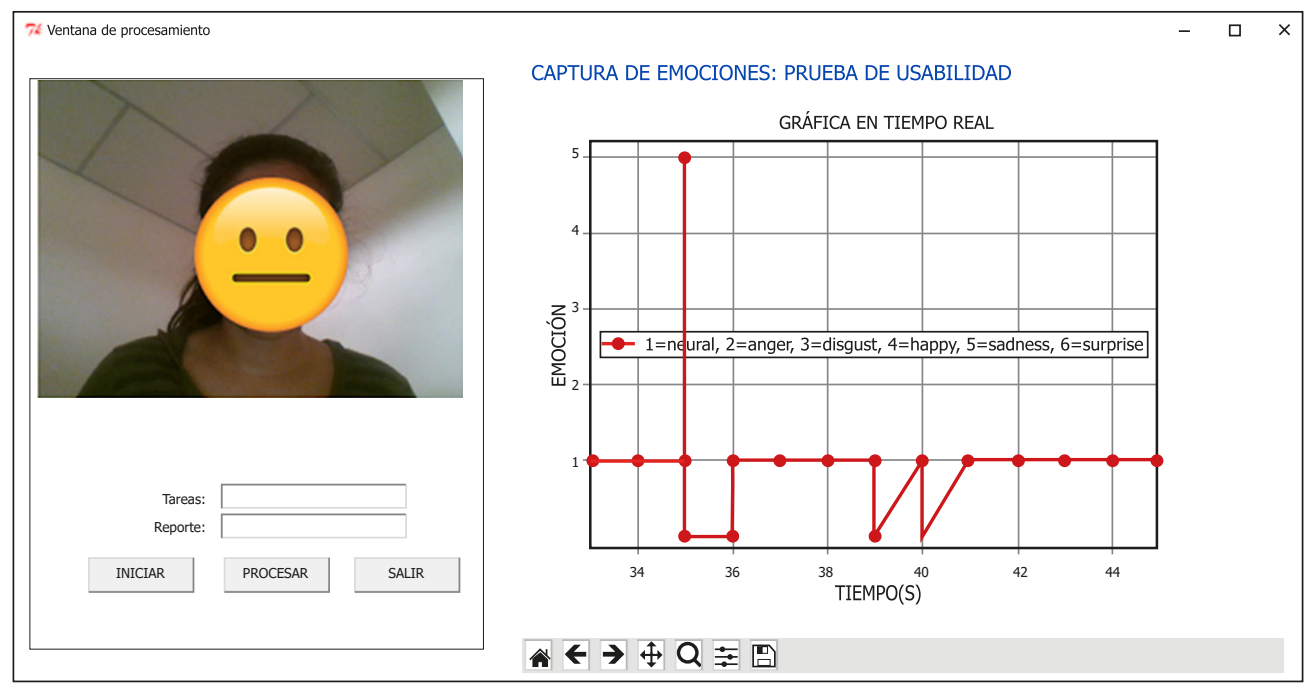

Figura 4. Predicción de emociones

Fuente: elaboración propia

Una vez terminada la prueba, el coordinador puede realizar a través de la opción Procesar de la herramienta, una discriminación de las tareas, al diligenciar en el campo de texto Tareas los tiempos específicos de duración de cada una de las tareas de la prueba, separando cada una mediante el símbolo de punto y coma (;) como se observa en la figura 5.

Finalmente, el sistema software permite visualizar de manera gráfica el estado emocional del usuario bajo dos variables que son el tiempo y la emoción (ver figura 6).

Las gráficas obtenidas permiten al coordinador de la prueba realizar un seguimiento del comportamiento emocional del usuario mientras interactúa dentro del laboratorio de usabilidad con un prototipo software, discriminado por líneas de colores que diferencian cada tarea, como se muestra en la figura 6 y si lo desea poder exportarlo en formato CSV al presionar la opción de guardar. 


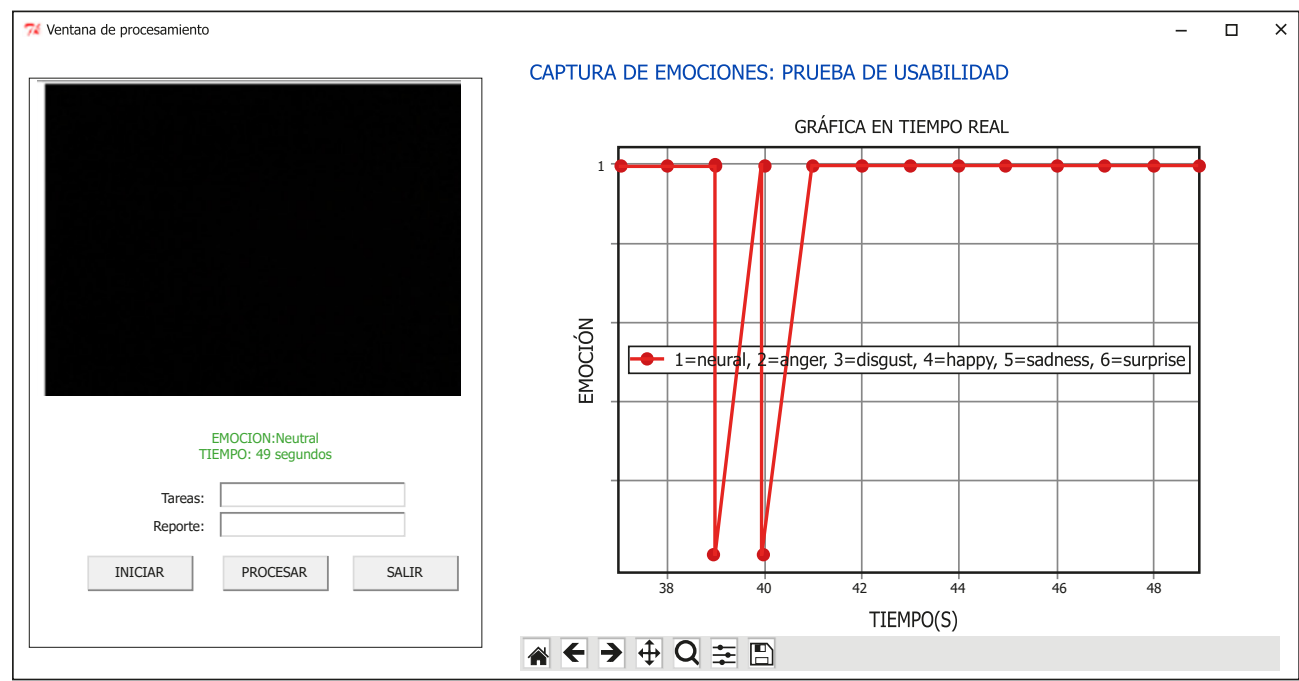

Figura 5. Configuración de tareas

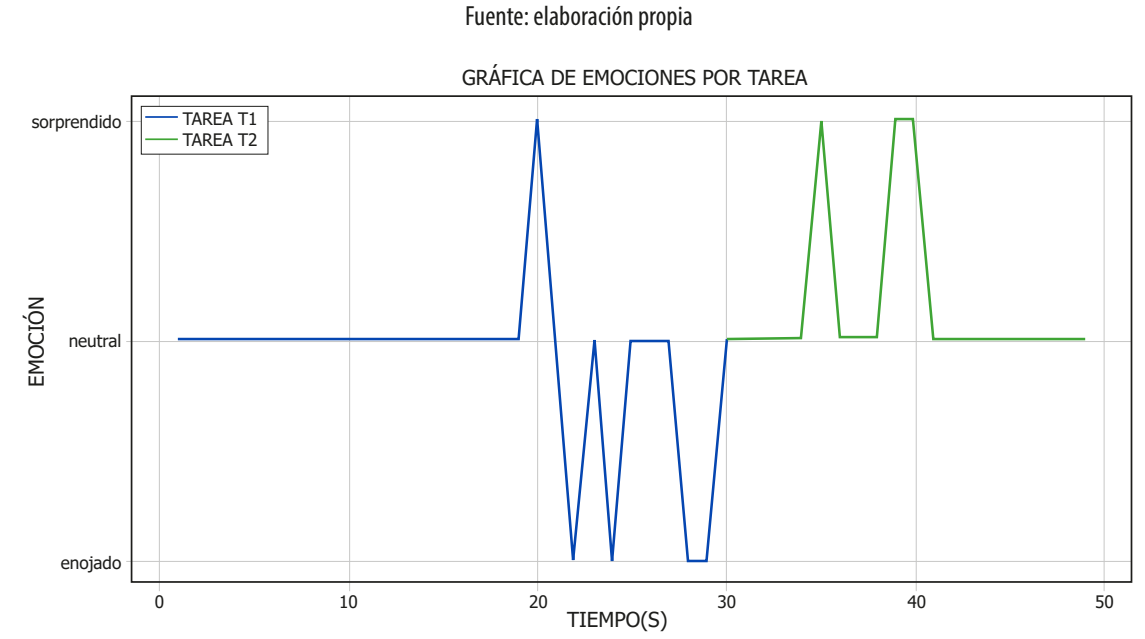

Figura 6. Generación de gráficas por tarea

Fuente: elaboración propia

\section{RESULTADOS}

En cuanto a la precisión de la detección de emociones realizadas por la herramienta dentro de la documentación de la librería OpenCV, se especifica que la predicción bajo condiciones adecuadas es superior al $70 \%$.

Las pruebas de tiempo de procesamiento y de consumo de memoria realizadas sobre el sistema software, fueron ejecutadas sobre una máquina física Lenovo con características de 8 GB de RAM y un procesador de cinco núcleos dentro de una 
versión de sistema operativo Windows 10 home. Para el desarrollo de las dos pruebas, se ejecutó el sistema software durante un tiempo arbitrario de 180 segundos, contando el número de predicciones que podía realizar, así como el tiempo de respuesta y la cantidad de memoria RAM empleada en cada predicción.

\subsection{Pruebas de tiempo de procesamiento}

En la figura 7 se evidencian los diferentes ciclos de procesamiento del sistema software durante un tiempo arbitrario de 180 segundos de una prueba hipotética, esto con el fin de verificar la pertinencia de la herramienta para realizar un seguimiento adecuado durante una prueba de usuario. El tiempo de procesamiento en la prueba alude al tiempo que tarda el sistema software en predecir una emoción del usuario. De acuerdo a la figura 8, en las primeras 68 invocaciones al método de predicción realizados por el sistema software, el tiempo de procesamiento oscila entre 35 y 182 milisegundos. A partir de la invocación 69 hasta la invocación 848 el tiempo de respuesta oscila entre 0 y 81,42 milisegundos. En este sentido el algoritmo de predicción emplea un tiempo inferior a los 100 milisegundos durante el $90 \%$ del tiempo que dura la prueba, lo que permite concluir que el sistema software tiene una de respuesta adecuada si se desea configurar para tomar una muestra del rostro del usuario cada segundo.

\section{Tiempo de procesamiento del módulo de predicción}

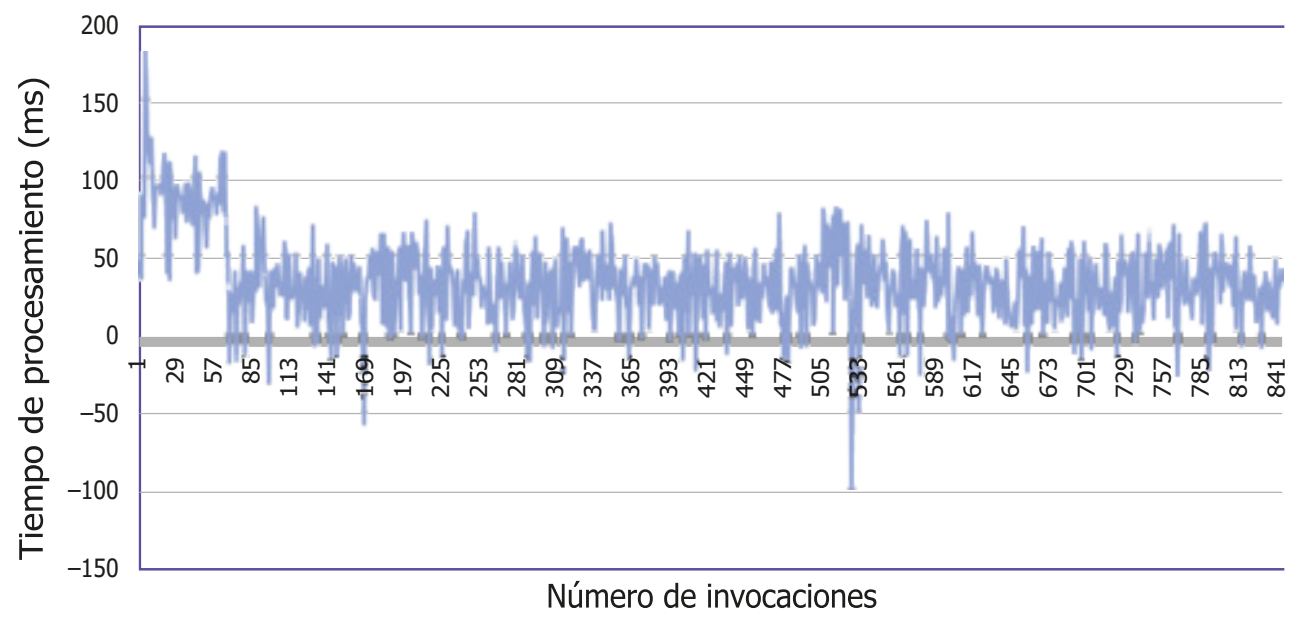

Figura 7. Tiempo de procesamiento del módulo de predicción

Fuente: elaboración propia

\subsection{Pruebas de consumo de memoria}

En la figura 8, se observan los diferentes consumos de memoria RAM durante un tiempo arbitrario de 180 segundos de una prueba hipotética. 
El consumo de memoria refiere la cantidad de memoria RAM que emplea el sistema software para predecir una emoción del usuario. De acuerdo con la figura 8, la cantidad de memoria RAM consumida por el módulo de predicción oscila entre 5 y $150 \mathrm{MB}$ a lo largo de toda la prueba, la mayoría de las veces tiene un valor cercano a los $100 \mathrm{MB}$.

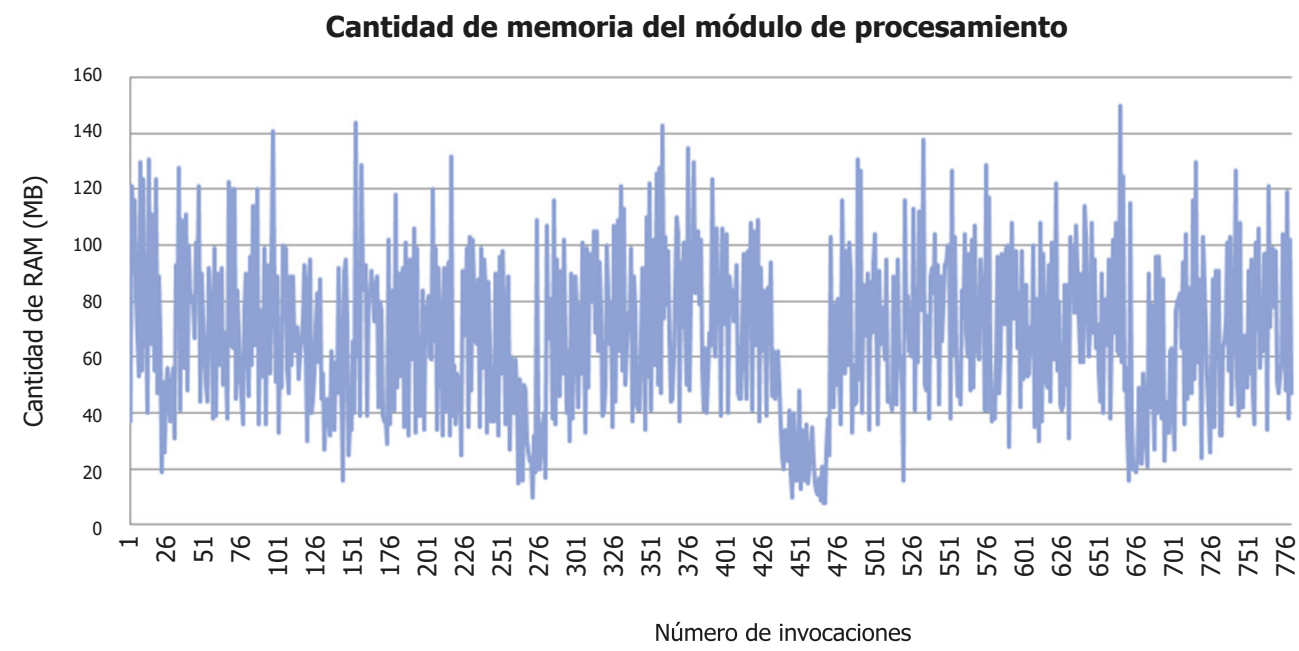

Figura 8. Cantidad de memoria RAM del módulo de predicción

Fuente: elaboración propia

Lo anterior permite concluir que el consumo de memoria RAM del sistema software es adecuado, teniendo en cuenta que correspondería a la sexta parte de un equipo con $1 \mathrm{~GB}$ de memoria RAM. En este sentido es adecuado para ser empleado en un laboratorio de usabilidad de equipos con características convencionales.

\section{CONCLUSIONES}

El análisis de la satisfacción a partir de la expresión facial del usuario es uno de los métodos menos intrusivos para la obtención de indicadores emocionales, comparado con el uso de sensores biométricos articulados al espacio corporal del usuario. Del mismo modo, la captura de la expresión facial del usuario aprovecha la cámara web que generalmente se utiliza en la habitación de usuario de un laboratorio de usabilidad.

El sistema software que permite realizar el análisis de la satisfacción, logra hacer el seguimiento al comportamiento emocional del sujeto en tiempo real durante una prueba ejecutada en un laboratorio de usabilidad, por lo que este aporte permite obtener indicadores del comportamiento emocional del usuario, de cara a la estimación objetiva de la satisfacción en pruebas de usabilidad. 
Las pruebas de tiempo de procesamiento y de consumo de memoria permiten concluir que el sistema software usado para conducir el estudio de la satisfacción en pruebas de usabilidad es adecuado para ser empleado en equipos con características convencionales, como los usados por un evaluador dentro de un laboratorio de usabilidad.

La propuesta realizada en este artículo contó con las ventajas provistas por la librería OpenCV, la cual admite dentro del sistema software desarrollado realizar las funciones de: captura, procesamiento y predicción de emociones. Las predicciones almacenadas y el tiempo en el que estas ocurren permiten un procesamiento de los datos para la graficación del comportamiento emocional, posibilitando al evaluador de la prueba comparar las interacciones que realiza el usuario con los estados emocionales que este manifiesta en las diferentes tareas.

Adicionalmente el sistema software propuesto posibilita, una vez terminada la prueba, diferenciar el comportamiento emocional por tareas, de modo que el evaluador puede segmentar a prueba de acuerdo con el número de tareas realizadas y el tiempo empleado por el usuario en cada una de ellas.

Como trabajo futuro derivado de la presente investigación, se busca mejorar este sistema software con la inclusión de otro tipo de datos estadísticos útiles para el evaluador, como el porcentaje de cada una de las emociones a lo largo de un test de usuario. Adicionalmente, se pretende disponer la herramienta dentro del laboratorio de usabilidad, adaptándola al contexto de los dispositivos SBC, de tal modo que pueda ser más portable. Igualmente, se aspira mejorar el modo en el que se configuran las diferentes tareas de la prueba, para que la interfaz de la herramienta resulte más intuitiva facilitando la tarea del coordinador.

\section{REFERENCIAS}

[1] J. Gonzáles, Jugabilidad y Videojuegos: Análisis y Diseño de la Experiencia del Jugador en Sistemas Interactivos de Ocio Electrónico, Beau Bassin, Mauricio: Editorial Académica Española, 2011.

[2] H. Alarcón, A. Hurtado, C. Pardo, C. Collazos y F. Pino, "Integración de técnicas de usabilidad y accesibilidad en el proceso de desarrollo de software de las mipymes", Avances en Sistemas e Informática, vol. 4, n. ${ }^{\circ}$ 3, pp. 149-156, 2007.

[3] Aenor, Requisitos ergonómicos para trabajos de oficina con pantallas de visualización de datos, UNE-EN ISO 9241-11, 1998.

[4] J. Enríquez y S. Casas, "Usabilidad en Aplicaciones Móviles”, Revista Informe Científico Técnico UNPA, vol. 5, n. ${ }^{\circ}$ 2, pp. 25-47, 2013. 
[5] G. Chanchí, M. Sánchez, A. Duque y W. Campo, "Propuesta de una herramienta para el análisis del estrés mental en pruebas de usabilidad", presentado en IV Jornadas de Interacción Humano Computador, Popayán, 2018.

[6] G. Chanchí, C. Muñoz y M. Camacho, "Experimentos de Usabilidad en IUnimayor”, Revista Almenara, ed. n. ${ }^{\circ} 14$, pp. 4-7, 2015.

[7] N. Botta, "Usabilidad en sitios web accesibles," Chacabuco: Universidad Abierta Interamericana, 2014.

[8] Real Academia Española, "Emociones," [En línea]. Disponible en https://dle.rae.es/ emoci\%C3\%B3n?m=form

[9] D. Papalia, S. Wendkos y R. Duskin, Psicología del desarrollo, 11 ed. Ciudad de México: Mc Graw Hill, 2009.

[10] A. Peinador, "Smartphone como soporte para interacción afectiva: expresión afectiva,”, [En línea]. Disponible en https://repositorio.uam.es/handle/10486/677160

[11] A. Fujita y R. Revetría, New Trends in Software Methodologies, Tools and Techniques, Amsterdam: IOS Press, 2012.

[12] A. Fernández y M. M. C. Dufey, "Expresión y reconocimiento de emociones: un punto de encuentro entre evolución, psicofisiología y neurociencias", Revista Chilena de Neuropsicología, vol. 2, n. ${ }^{\circ}$ 1, pp. 8-20, 2007.

[13] A. Ardil, "El lenguaje no verbal. Claves culturales para la competencia comunicativa e intercultura," Tesis de Master, Universidad de Oviedo, Oviedo, 2016.

[14] J. Díaz y E. Flores, "La estructura de la emoción Humana: un modelo cromático del Sistema afectivo", Revista Salud Mental, vol. 24, n. . 4, pp. 20-35, 2001.

[15] E. Marin, “Detección de emociones del usuario,”Trabajo de grado, Pontificia Universidad Católica de Valparaíso,Valparaíso, 2014.

[16] P. Rodríguez, "Método automatizado para la evaluación de la usabilidad en sistemas elearning usando monitoreo de actividad cerebral," Tesis de doctorado, Universidad Nacional, Medellín, 2015. 